\title{
Debonding behavior and interfacial stress in carbon fiber-reinforced polymer plate-strengthened top flange of box-girder bridge
}

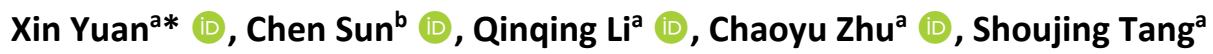 \\ a School of Civil Engineering, Suzhou University of Science and Technology, Suzhou, 215011, P. R. China. Email: yuanxin9988@163.com, \\ liqinqing201906@163.com zhuchaoyu@126.com, tangshoujing2020@163.com. \\ ${ }^{b}$ School of Science, Nanjing University of Science and Technology, Nanjing, 210094, P.R. China. Email:kevin6s6@163.com
}

* Corresponding author

https://doi.org/10.1590/1679-78256271

\begin{abstract}
The interfacial stress in a carbon fiber-reinforced polymer (CFRP) plate for strengthening the top flange of a box-girder bridge was theoretically investigated. According to the force analysis results of the box-girder structure, the most unfavorable force area was selected for modeling analysis. Using the simplified beam model, a CFRP plate-concrete interfacial-stress calculation model was derived, and the closed-form solution of the calculation model was obtained. Equivalent simplified test specimens were designed according to the box-girder structure to verify the reliability of the theoretical model. Then, four-point bending tests of the sample were performed. In these tests, the debonding failure modes and strain in the CFRP plate were examined. The CFRP plate-concrete interfacial stress was calculated according to the strain data of the CFRP plate in the experimental tests. Then compared the experimental results with the theoretical results. Furthermore, the corresponding interfacial stresses were calculated and compared according to the different load levels specified in the code. A series of theoretical and experimental comparisons were performed to verify the reliability of the proposed CFRP-concrete interfacial stress closed-form solution model.
\end{abstract}

\section{Keywords}

CFRP plate, strengthened box girder, debonding failure, interfacial stresses, theoretical model, bridge

\section{Graphical Abstract}
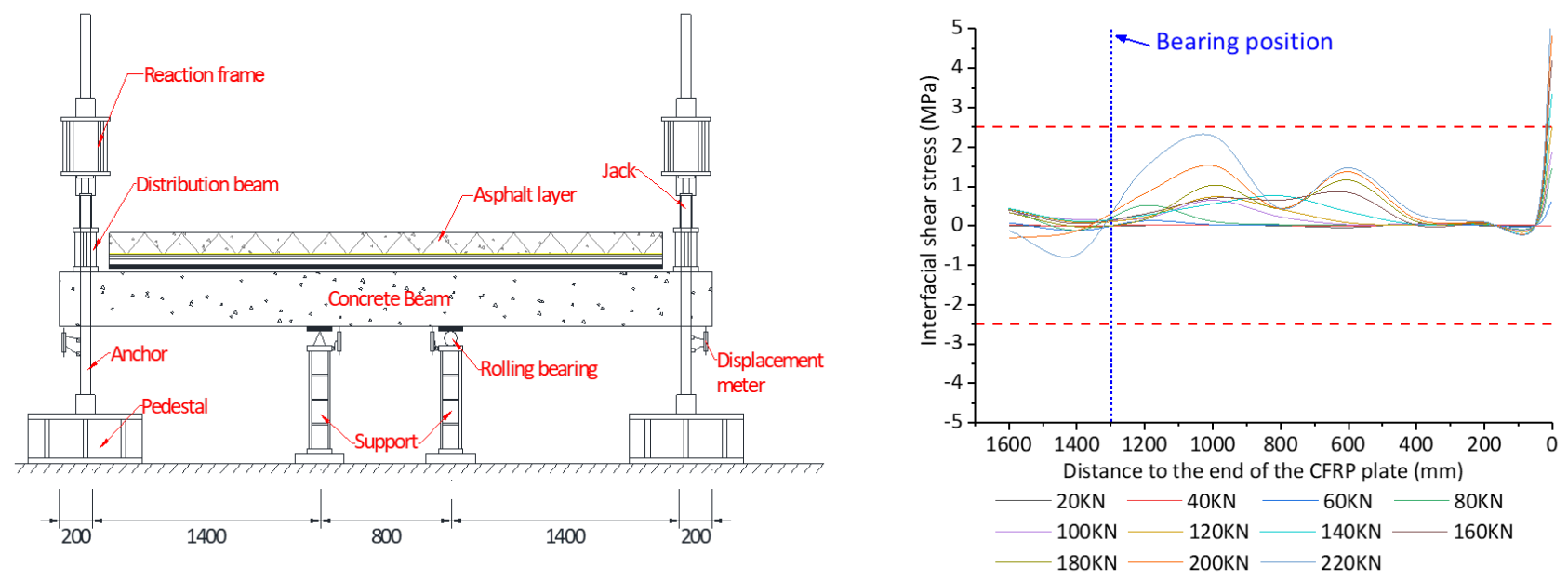

Received September 22, 2020. In revised form March 22, 2021. Accepted March 24, 2021. Available online March $29,2021$. https://doi.org/10.1590/1679-78256271

Latin American Journal of Solids and Structures. ISSN 1679-7825. Copyright @ 2021. This is an Open Access article distributed under the terms of the Creative Commons Attribution License, which permits unrestricted use, distribution, and reproduction in any medium, provided the original work is properly cited. 


\section{INTRODUCTION}

Carbon fiber-reinforced polymer (CFRP) is generally formed through a series of heat treatments, and carbon fiber tow and epoxy resin are typically used as basic materials. Kromoser et al. (2019) introduced CFRP as a lightweight and high-strength material whose density and tensile strength are approximately $20 \%$ of and five times those of steel, respectively; the CFRP plate shape is shown in Figure 1(a). Burgoyne (2004) reported that because of its excellent material properties, CFRP has been used to enhance the durability of reinforced concrete structures. Compared with the traditional bridge-strengthening methods, the application of CFRP materials can significantly reduce the dead weight and impact on the bridge structure. Czaderski \& Motavalli (2007) used a prestressed CFRP plate to strengthen an old concrete bridge and employed the gradient method to anchor the plate. Hosseini et al. (2009) performed a series of truck-loading tests on a non-prestressed CFRP-strengthened metallic bridge and concluded that the tensile stresses were reduced via strengthening. Gallego et al. $(2017,2018)$ studied the lateral bending performance of a concrete box-girder bridge and proposed the use of a CFRP board to strengthen the box-girder cantilever.

Different types of cracks appear in concrete box-girder bridges when they are subjected to various loads and exposed to harsh environments. In this regard, the use of the CFRP plate can improve the lateral rigidity of the girder. The commonly adopted method involves pasting a CFRP plate of a certain length on the top edge of the concrete box girder, as shown in Figure 1(b). In the negative-flexural moment zone, the CFRP plate can maintain the tensile force by functioning similar to that of tensile steel bars. However, the debonding failure of the CFRP plate usually occurs at the end of the concrete structure reinforced by the CFRP plate, as shown in Figure 1(b).

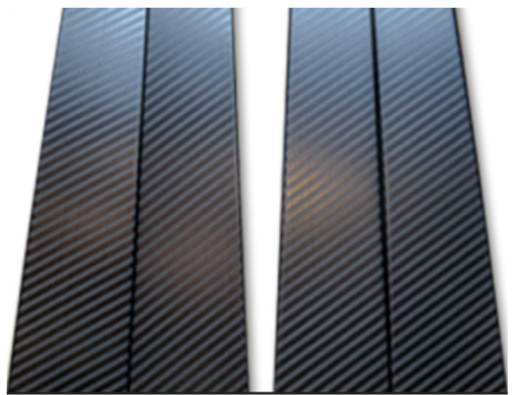

(a) CFRP plate

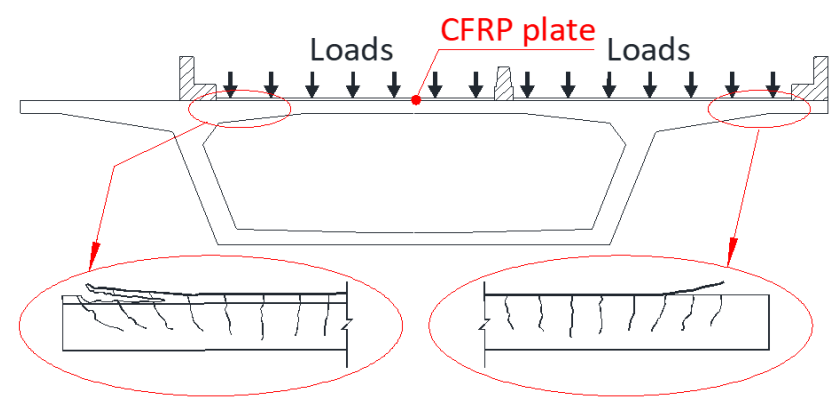

(b) strengthen method and debonding failure modes

Figure 1: CFRP plate reinforcement scheme and plate end debonding failure.

Lu et al. (2005) and Smith \& Teng (2002) investigated the main cause of the CFRP plate-end debonding failure and found that the interfacial stress between the CFRP and the concrete was larger than the ultimate bearing capacity of the concrete. Sun et al. $(2017 \mathrm{a}, 2017 \mathrm{~b})$ simulated the entire debonding failure process of a CFRP plate on a concrete structure and proposed a bi-fold line interfacial bonding model based on finite-element analysis results. Ferrier et al. $(2011,2016)$ and Ferrier and Agbossou (2017) performed a series of experimental studies on FRP concrete double-shear specimens (test temperature range of -40 to $20^{\circ} \mathrm{C}$ ). The interface characteristics of CFRP concrete after special process treatment at a certain temperature were analyzed using a high-order fracture mechanics model. Wang \& Petrů (2019) experimentally investigated the mechanical behavior of the CFRP-concrete interface after freeze-thaw cycling. The results indicated that the failure mode was affected by the freeze-thaw cycling; as the number of cycles increased, the failure mode changed from tensile failure to interface failure.

In this study, the top flange of a box-girder bridge was strengthened using a CFRP plate to investigate the facile occurrence of plate-end debonding failure. Using the force analysis results of the box girder, according to the debonding failure of the plate end of the CFRP-concrete interface, a closed-form theoretical calculation model for the interfacial stress was proposed. Beam specimens were designed according to the equivalent simplification of the box girder. A fourpoint flexural test was performed on the test beams, and the reliability of the proposed calculation model was verified by comparing experimental results with theoretical results.

In this paper, a calculation method for the higher-order analytical solutions of interfacial stress to avoid the closedform approximate solution of a simply supported beam is presented. Moreover, different loading situations, including a flexural moment, uniform load, and concentrated load, are simultaneously considered in the proposed calculation model. One of the advantages of the model is that it derives the expression of the solution in a closed form. Moreover, in the thickness direction of the adhesive layer, the longitudinal stress variation of the interfacial stress is considered. 


\section{THEORETICAL MODEAL OF INTEEFACIAL STRESS}

\subsection{Analysis model}

Lateral force analysis of the box girder is performed according to the actual loading condition of the bridge structure shown in Figure 2(a). According to the mechanical analysis results, the maximum negative flexural moment exists on one side of the box-girder roof. Thus, this part of the box-girder roof is selected for the analysis; the simplified beam model is shown in Figure 2(b). Considering that the box-girder web has a certain thickness, it can be simplified as a support for the box-girder roof. The dead weight of the asphalt pavement on the bridge deck is regarded as a uniform load (q). To simulate the actual forces on the structure, flexural moments and concentrated loads are applied to the simplified model.

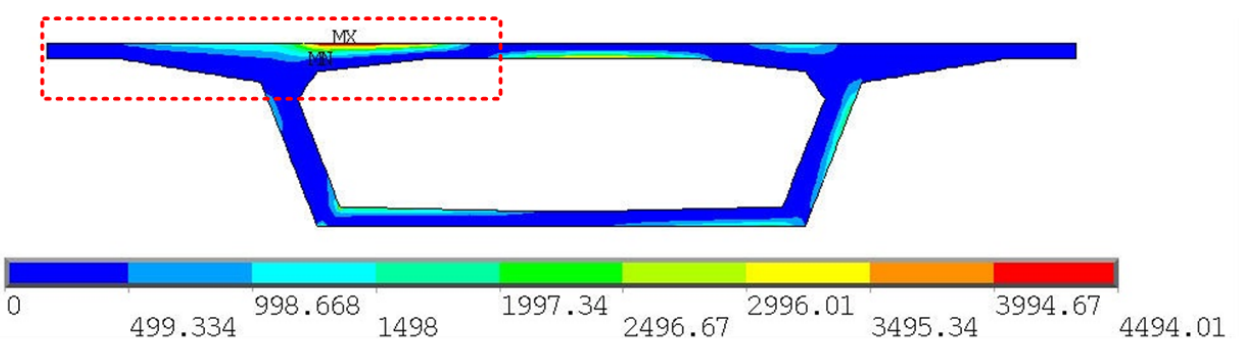

(a) Analysis results for the lateral force of the box girder

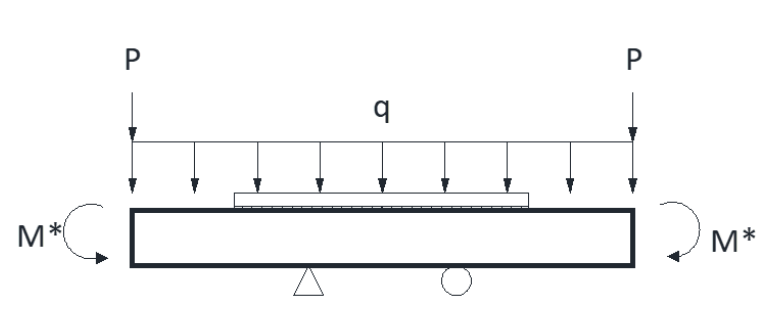

(b) Simplified beam model

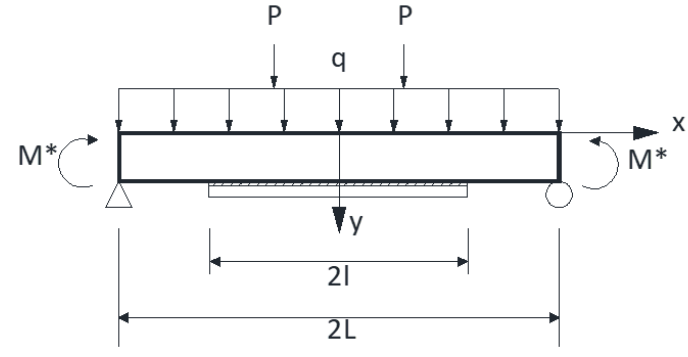

(c) Equivalent simply supported beam model

Figure 2: Equivalent conversion process of the theoretical model.

To facilitate its use in actual engineering, the simplified beam model is subjected to further equivalent transformation (Figure 2(b)). In the equivalent simply supported beam model shown in Figure 2(c), a coordinate system wherein the origin is the intersection of the box-girder web and roof is established. The plane where the top flange is regarded as the X-axis, and the positive direction of the Y-axis points to the bottom plate of the box girder. In this equivalent calculation model, the CFRP plate originally placed on the top flange of the box girder is transferred to the bottom of the beam model. The lengths of the beam and CFRP plate are $2 L$ and 2 , respectively.

According to the principle of equivalent force, the original simple support located at the midspan is converted into concentrated loads and placed at the beam ends. After the conversion, an equivalent simply supported beam model with traditional simple support boundary conditions is obtained. The original reaction forces of the midspan support are converted into two new concentrated forces $(P)$, which are loaded on the both sides of the midspan position of the beam model. Moreover, the concentrated load originally located at the beam end, as shown in Figure 2(b), is converted into a simple support, as shown in Figure 2(c). According to the force analysis results, the actual force in the beam remains unchanged before and after the transition at the boundary condition.

\subsection{Basic assumptions}

The theoretical model is subjected to a flexural moment (M), concentrated load (P), and uniform load (q). In the interfacial stress analysis of this model, the following basic assumptions are made.

1. The beam model is divided into several layers, each of which is considered as linearly elastic. The anisotropy of the material is ignored, and the material is considered to be homogeneous.

2. The deformation of adjacent layers at their interface is ignored; i.e., it is assumed that there is no relative slip at the CFRP-concrete interface. 
3. The CFRP plate and concrete beam in the model follow the Euler beam theory, and the adhesive layer conforms to the plane stress-strain criterion.

4. The thin-plate flexural theory is used for the CFRP plate-reinforced concrete beam.

5. The longitudinal stress in the thickness direction of the adhesive layer is assumed to change linearly.

6. For a beam with debonding failure, the shear deformation is presumed to cause the anisotropy of the layers at the interface in the longitudinal section of the beam.

\subsection{Boundary conditions}

For modeling convenience, the concrete beams, bonding layers, and CFRP plates are labeled with the numbers 1-3, as shown in Figure 3(a). Similarly, each interface layer is marked with a number ranging from (0) to (3). For the beam model cross section (Figure $3(b)$ ), the width of each interface layer is marked as $b(i)$, where $i$ is the number of the interface layer. Moreover, the width of the adhesive layer is considered to be identical to that of the CFRP plate, i.e., $b(0)=b(1)=b(2)$.

Each layer is separately analyzed according to the established coordinate system in the overall beam model. A local coordinate system can be established at the geometric center of different layers, as shown in Figure 3(c). The force condition of selected beam microsegments based on which the force-balance equations below are established is presented in Figure 3(c).

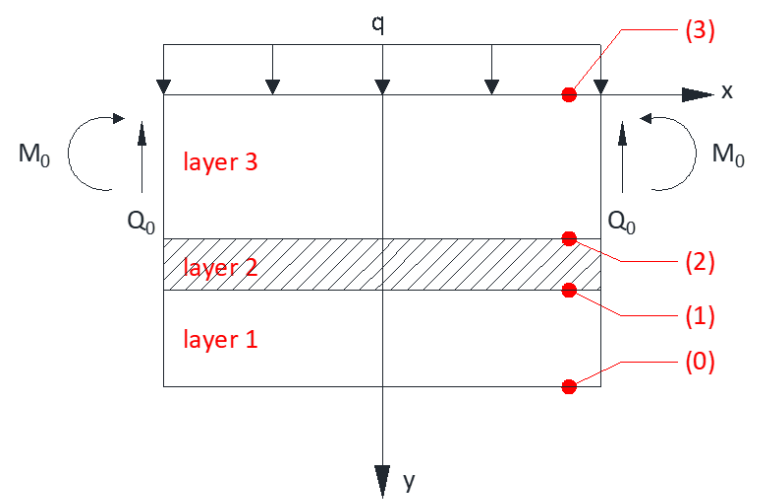

(a) Beam segment

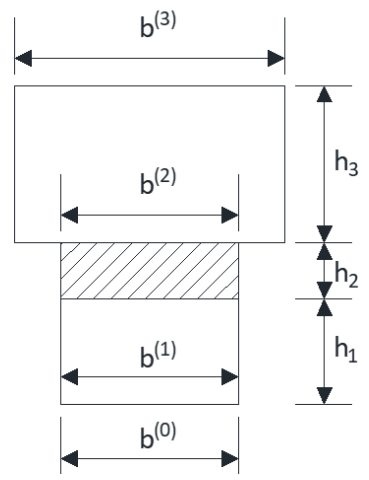

(b) Cross section

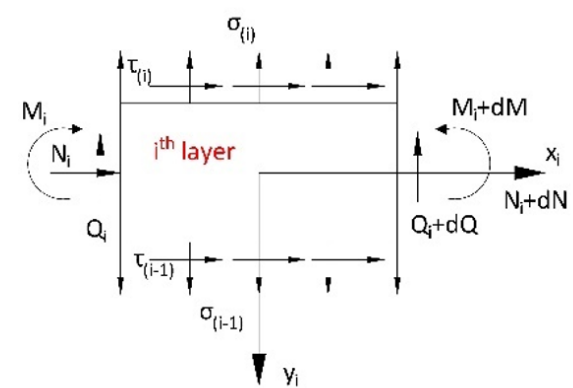

(c) Force analysis of the $i^{\text {th }}$ layer

Figure 3: Force analysis of the proposed model.

Axial-force equilibrium:

$\frac{d N_{i}}{d x}=b^{(i-1)} \tau_{(i-1)}-b^{(i)} \tau_{(i)}$.

Shear-force equilibrium:

$\frac{d Q_{i}}{d x}=b^{(i-1)} \sigma_{(i-1)}-b^{(i)} \sigma_{(i)}-q$.

Moment equilibrium:

$\frac{d M_{i}}{d x}=Q_{i}-\frac{h_{i}}{2}\left(b^{(i-1)} \tau_{(i-1)}-b^{(i)} \tau_{(i)}\right)$

The definitions of the symbols in the foregoing equations are presented in Table 1. 
Table 1: Symbols used in the equations.

\begin{tabular}{cccc}
\hline Symbol & Meaning & Symbol & Meaning \\
\hline$N_{i}$ & axial force & $\sigma_{(i)}$ & normal stresses in the $\mathrm{i}^{\text {th }}$ layer surface \\
$Q_{i}$ & shear force & $b^{(i)}$ & width of the $\mathrm{i}^{\text {th }}$ layer \\
$M_{i}$ & moment in the $\mathrm{i}^{\text {th }}$ layer surface & $h^{(i)}$ & height of the $\mathrm{i}^{\text {th }}$ layer \\
$\tau_{(i)}$ & shear stresses in the $\mathrm{i}^{\text {th }}$ layer surface & & \\
\hline
\end{tabular}

Attentionally, $q$ is equal to the uniform load when $\mathrm{i}=3$; otherwise, $q=0$.

The following equations can be established according to the boundary conditions shown in Figure 3(c):

$x= \pm l$

$N_{i}=0$

$Q_{i}=\left\{\begin{array}{lll}0 & , & i \neq 3 \\ q l+p & , & x \leq l^{\prime}, i=3 \\ q l & , & l^{\prime} \leq x \leq L, i=3\end{array}\right.$

$M_{i}= \begin{cases}0 & , \quad i \neq 3 \\ M^{*}+\frac{q\left(L^{2}-x^{2}\right)}{2}+p(L-x) & , \quad-l^{\prime} \leq x \leq l^{\prime}, i=3 \\ M^{*}+\frac{q\left(L^{2}-x^{2}\right)}{2}+p\left(L-l^{\prime}\right) & , \quad-L \leq x \leq-l^{\prime} \text { or } l^{\prime} \leq x \leq L, i=3\end{cases}$

where $l$ and $L$ represent the lengths of the concrete beam and CFRP plate, respectively, and $M^{*}$ represents the moment in the $\mathrm{i}^{\text {th }}$ layer.

\subsection{Analytical solution}

For the CFRP plate-adhesive interface $(i=1)$ and the adhesive-concrete interface $(i=2)$, the interfacial stress can be calculated using the Fourier series expansion equations. The normal and shear stresses in the $i^{\text {th }}$ layer can be expressed as follows:

$$
\begin{gathered}
\sigma_{(i)}=\sum_{m=1} a_{m}^{(i)} \cos \frac{m \pi x}{l} \\
\tau_{(i)}=\sum_{m=1} b_{m}^{(i)} \sin \frac{m \pi x}{l} .
\end{gathered}
$$

By substituting Eq. (3) in Eq. (1), the following equations can be derived: 


$$
\begin{aligned}
& N_{i}=\sum_{m=1}\left(\frac{l}{m \pi}\right)\left(b^{(i)} b_{m}^{(i)}-b^{(i-1)} b_{m}^{(i-1)}\right)\left(\cos m \pi-\cos \frac{m \pi x}{l}\right) \\
& Q_{i}=\sum_{m=1}\left(\frac{l}{m \pi}\right)\left(b^{(i)} a_{m}^{(i)}-b^{(i-1)} a_{m}^{(i-1)}\right) \sin \frac{m \pi x}{l}+ \begin{cases}0, & i \neq 3 \\
-q x & , \quad i=3\end{cases} \\
& M_{i}=\sum_{m=1}\left(\frac{l}{m \pi}\right)\left[\frac{l}{m \pi}\left(b^{(i)} a_{m}^{(i)}-b^{(i-1)} a_{m}^{(i-1)}\right)-\frac{h_{i}}{2}\left(b^{(i)} b_{m}^{(i)}+b^{(i-1)} b_{m}^{(i-1)}\right)\right] \\
& \left(\cos m \pi-\cos \frac{m \pi x}{l}\right) \\
& + \begin{cases}0 & , i \neq 3 \\
M^{*}+\frac{q\left(L^{2}-x^{2}\right)}{2}+p(L-x) & , i=3,-l^{\prime} \leq x \leq l^{\prime} \\
M^{*}+\frac{q\left(L^{2}-x^{2}\right)}{2}+p\left(L-l^{\prime}\right) & , i=3 \quad,-L \leq x \leq-l^{\prime} \text { or } l^{\prime}<x \leq L\end{cases}
\end{aligned}
$$

where $a_{m}^{(i)}$ and $b_{m}^{(i)}$ are considered to be zero when i is equal to 0 and 3 , respectively. No adjacent interfaces are connected to the upper surface of the concrete beam or the lower surface of the CFRP plate; therefore, there is no interfacial stress on these two surfaces.

For the adhesive layer, considering assumptions (3) and (5), the following equations may be satisfied:

$$
\begin{aligned}
& \frac{\partial \sigma_{x}^{(2)}}{\partial x}+\frac{\partial \sigma_{x y}^{(2)}}{\partial y}=0 \\
& \frac{\partial \sigma_{x y}^{(2)}}{\partial x}+\frac{\partial \sigma_{y}^{(2)}}{\partial y}=0
\end{aligned}
$$

where $\sigma_{x}^{(i)}$ and $\sigma_{y}^{(i)}$ represent the normal stresses in the horizontal and vertical directions of the $\mathrm{i}^{\text {th }}$ layer, respectively, and $\sigma_{x y}^{(i)}$ represents the normal stress in the plane corresponding to $\sigma_{x}^{(i)}$ and $\sigma_{y}^{(i)}$.

Then, the following equations can be obtained.

$$
\begin{aligned}
& \sigma_{x}^{(i)}= \frac{1}{\chi_{i}}\left\{\frac{\xi_{i}-\xi_{i-1}}{h_{i}}-\frac{12 y_{i}}{h_{i}^{3}}\left[\frac{h_{i}}{2}\left(\xi_{i}+\xi_{i-1}\right)+\eta_{i-1}-\eta_{i}\right]\right\} \\
& \cdot \sum_{m=1}\left(\frac{1}{m \pi}\right)^{2} a_{m}^{(1)}\left(\cos m \pi-\cos \frac{m \pi x}{l}\right)+ \begin{cases}0 & , \quad i=1 o r 2 \\
\sigma_{x}^{a}, & i=3\end{cases} \\
& \sigma_{y}^{(i)}= \begin{cases}0 & , i=1 \text { or } 3 \\
\xi_{i}\left(y_{2}\right) \sum_{m=1} a_{m}^{(1)} \cos \frac{m \pi x}{l}, & i=2\end{cases}
\end{aligned}
$$




$$
\begin{aligned}
\tau_{x y}^{(i)}= & \frac{1}{\chi_{i}}\left\{\chi_{i} \xi_{i-1}+\frac{1}{h_{i}}\left(\xi_{i}-\xi_{i-1}\right)\left(\frac{h}{2}-y_{i}\right)\right. \\
& \left.+\frac{6}{h_{i}^{3}}\left[\left(\eta_{i}-\eta_{i-1}\right)-\frac{h_{i}}{2}\left(\xi_{i}+\xi_{i-1}\right)\right]\left(\frac{h_{i}^{2}}{4}-y_{i}^{2}\right)\right\} \\
& \cdot \sum_{m=1}\left(\frac{1}{m \pi}\right) a_{m}^{(1)} \sin \frac{m \pi x}{l}+ \begin{cases}0 & , i=1 \text { lor } 2 \\
\tau_{x}^{a}, & i=3\end{cases}
\end{aligned}
$$

In the foregoing, $\chi_{i}$ can be expressed as $\chi_{i}=\frac{b^{(i)}}{b^{(1)}}$, where $b^{(i)}$ and $b^{(1)}$ represent the widths of the $\mathrm{ith}^{\text {th }}$ and first layer interfaces, respectively. Moreover, $\xi_{i}$ and $\eta_{i}$ are parameters related to the interface size and can be expressed as $\xi_{i}=\frac{m \pi}{l} \chi_{i} \frac{b_{m}^{(i)}}{a_{m}^{(1)}}$ and $\quad \eta_{i}=\chi_{i} \frac{a_{m}^{(i)}}{a_{m}^{(1)}}$, respectively. $\xi_{1}\left(y_{2}\right)$ is a simplified index that can be expressed as follows:

$$
\begin{aligned}
\xi_{1}\left(y_{2}\right)= & \frac{1}{\chi_{2}}\left\{\chi_{2}+\left[\frac{3}{2 h_{2}}\left(\eta_{2}-1\right)+\xi_{1}-\frac{1}{4}\left(\xi_{2}+5 \xi_{1}\right)\right] \cdot\left(\frac{h_{2}}{2}-y_{2}\right)\right. \\
& -\frac{1}{2 h_{2}}\left(\xi_{2}-\xi_{1}\right)\left(\frac{h_{2}^{2}}{4}-y_{2}^{2}\right) \\
& \left.-\left[\frac{2}{h_{2}^{3}}\left(\eta_{2}-1\right)+\frac{1}{h_{2}^{2}}\left(\xi_{2}+\xi_{1}\right)\right]\left(\frac{h_{2}^{3}}{8}-y_{2}^{2}\right)\right\}
\end{aligned}
$$

Moreover, the following relationships can be obtained:

$$
\begin{aligned}
& \xi_{i}=\xi_{i-1} \frac{\theta_{i} h_{i}}{\theta_{1} h_{1}} \frac{\sum_{j=1}^{i} h_{j}-\frac{h_{i}}{2}-h_{0}}{\frac{h_{1}}{2}-h_{0}} \xi_{1} \\
& \eta_{i}=\eta_{i-1}+\frac{h_{i}}{2}\left(\xi_{i}+\xi_{i-1}\right)-\frac{\theta_{i} h_{i}^{3}}{12 \theta_{1} h_{1}\left(\frac{h_{i}}{2}-h_{0}\right)} \xi_{1},
\end{aligned}
$$

where ${ }^{\theta_{i}}$ represents the elastic-modulus ratio of the $\mathrm{i}^{\text {th }}$ layer interface to the first layer interface. It can be expressed as $\theta_{i}=\chi_{i} \frac{E_{11}^{(i)}}{E_{11}^{(1)}}$

thicknesses and can be expressed as

$$
h_{0}=\frac{1}{A_{0}} \sum_{i=1}^{3}\left(\theta_{i} h_{i}\left(\sum_{j=1}^{i} h_{j}-\frac{h_{i}}{2}\right)\right) \text {, where } A_{0}=\sum_{i=1}^{3} \theta_{i} h_{i}
$$
. In practice, when $\mathrm{i}=1, \eta_{i}$ is considered to be equal to 1 , and when $\mathrm{i}=0$ or $3, \xi_{i}$ and $\eta_{i}$ are regarded as 0 .

By substituting Eqs. (4)-(8) in Eq. (3), all the variables in Eq. 3-except for $a_{m}^{(i)}$-can be expressed in terms of the other known variables. According to the principle of minimum total complementary energy, the value of $a_{m}^{(i)}$ can be 
obtained. Considering the structural symmetry of the beam model, half of the beam structure is selected for analysis, and the following potential energy relationship is established.

$$
\begin{aligned}
U & =\frac{b^{(1)}}{2} \sum_{i=1}^{3} \chi_{i} \int_{-\left(h_{i} / 2\right)}^{+\left(h_{i} / 2\right)} \int_{0}^{l}\left[\frac{1}{E_{11}^{(i)}} \sigma_{x}^{(i)} \sigma_{x}^{(i)}+\frac{1}{E_{22}^{(i)}} \sigma_{y}^{(i)} \sigma_{y}^{(i)}\right. \\
& \left.-\frac{2 v_{12}^{(i)}}{E_{11}^{(i)}} \sigma_{x}^{(i)} \sigma_{y}^{(i)}+\frac{1}{G_{12}^{(i)}} \tau_{x y}^{(i)} \tau_{x y}^{(i)}\right] d x d y
\end{aligned}
$$

According to the principle of the variational method, the following equations can be obtained from Eq. (9):

$S_{1}\left(\frac{l}{m \pi}\right)^{2} \cos m \pi \sum_{m=1}\left(\frac{l}{m \pi}\right)^{2} a_{m}^{(1)} \cos m \pi+S_{2}\left(\frac{l}{m \pi}\right)^{4} a_{m}^{(1)}$
$+S_{3}\left(\frac{l}{m \pi}\right)^{2} a_{m}^{(1)}+S_{4} a_{m}^{(1)}=S_{5}\left(\frac{l}{m \pi}\right)^{2} \cos m \pi+S_{6}\left(\frac{l}{m \pi}\right)^{4} \cos m \pi$

where the parameters $S_{1}, S_{2}, S_{3}, S_{4}, S_{5}$, and $S_{6}$ are expressed as follows:

$S_{1}=\sum_{i=1}^{3} \frac{b^{(1)}}{\chi_{1} E_{11}^{(i)}}\left\{\frac{\left(\xi_{i}-\xi_{i-1}\right)^{2}}{h_{i}}+\frac{12}{h_{i}^{3}}\left[x \frac{h_{i}}{2}\left(\xi_{i}+\xi_{i-1}\right)+\eta_{i-1}-\eta_{i}\right]^{2}\right\}$

$S_{2}=\frac{S_{1}}{2}$

$$
\begin{aligned}
S_{3}= & \sum_{i=1}^{3} \frac{b^{(1)} h_{i}}{\chi_{i} G_{12}^{(i)}}\left\{\xi_{i-1}^{2}\left[\frac{1}{2}\left(\frac{\chi_{i}}{\chi_{i-1}}-1\right)^{2}+\frac{1}{15}\right]\right. \\
& +\xi_{i-1}\left(\eta_{i}-\eta_{i-1}\right)\left[\frac{\chi_{i}}{\chi_{i-1} h_{i}}-\frac{11}{10 h_{i}}\right]+\frac{1}{15} \xi_{i}\left(\xi_{i}-\xi_{i-1}\right) \\
& \left.+\frac{1}{10 h_{i}} \xi_{i}\left(\eta_{i}-\eta_{i-1}\right)+\frac{3}{5 h_{i}^{2}}\left(\eta_{i}-\eta_{i-1}\right)^{2}\right\} \\
& -\frac{\mu_{12}^{(2)} b^{(1)} h_{2}}{15 \chi_{2} E_{11}^{(2)}} \cdot\left[2\left(\xi_{1}^{2}+\xi_{2}^{2}\right)+\xi_{1} \xi_{2}\left(14-15 \chi_{2}\right)\right. \\
& \left.+\frac{18}{h_{2}}\left(\xi_{1}+\xi_{2}\right)\left(1-\eta_{2}\right)+\frac{15 \chi_{2}}{h_{2}}\left(\xi_{1} \eta_{2}-\xi_{2}\right)+\frac{18}{h_{2}^{2}}\left(1-\eta_{2}\right)^{2}\right]
\end{aligned}
$$




$$
\begin{aligned}
S_{4} & =\frac{b^{(1)} h_{2}}{420 \chi_{2} E_{22}^{(2)}}\left\{h _ { 2 } ^ { 2 } \left[70\left(\chi_{2}-1\right)^{2} \xi_{1}^{2}+2\left(\xi_{1}+\xi_{2}\right)\left(\xi_{2}-6 \xi_{1}\right)\right.\right. \\
& \left.+14 \chi_{2} \xi_{1}^{2}+7\left(4-3 \chi_{2}\right) \xi_{1} \xi_{2}\right]+h_{2}\left[2\left(1-\eta_{2}\right)\left(11 \xi_{2}+67 \xi_{1}\right)\right. \\
& +7 \chi_{2} \xi_{1}\left(30 \chi_{2}+21 \eta_{2}-46\right)-35 \chi_{2} \xi_{2} \\
& \left.+\left[210 \chi_{2}\left(\chi_{2}+\eta_{2}-1\right)+78\left(\eta_{2}-1\right)^{2}\right]\right\}
\end{aligned}
$$

$$
\begin{aligned}
& \left\{\begin{array}{l}
\text { when }-l^{\prime} \leq x \leq l^{\prime}, \\
\frac{1}{\chi_{3} E_{11}^{(3)}} \frac{1}{h_{3}^{3}}\left[2 q\left(3 L^{2}-l^{2}\right)+12\left(M^{*}+p L\right)-3 p\left(L-l^{\prime}\right)\right]
\end{array}\right. \\
& S_{5}=\left\{\left[\frac{h_{3}}{2} \xi_{2}+\eta_{2}\right]+\frac{q}{\chi_{3} G_{12}^{(3)}}\left(\frac{6 \eta_{2}}{5 h_{3}}+\frac{11 \xi_{2}}{10}-\frac{\chi_{3} \xi_{2}}{\chi_{2}}\right)\right. \\
& \text { when }-L \leq x \leq-l^{\prime} \text { or } l^{\prime}<x \leq L \text {, } \\
& \frac{1}{\chi_{3} E_{11}^{(3)}} \frac{1}{h_{3}^{3}}\left[2 q\left(3 L^{2}-l^{2}\right)+12\left(M^{*}+p L-p l^{\prime}\right)\right] \\
& \cdot\left[\frac{h_{3}}{2} \xi_{2}+\eta_{2}\right]+\frac{q}{\chi_{3} G_{12}^{(3)}}\left(\frac{6 \eta_{2}}{5 h_{3}}+\frac{11 \xi_{2}}{10}-\frac{\chi_{3} \xi_{2}}{\chi_{2}}\right)
\end{aligned}
$$

$S_{6}=\frac{1}{\chi_{3} E_{11}^{(3)}} \frac{1}{h_{3}^{3}} \frac{12 q}{h_{3}^{3}}\left(\frac{h_{3}}{2} \xi_{2}+\eta_{2}\right)$.

To further simplify Eq. (10), $S_{0}, \alpha$, and $\beta$ are introduced. The simplified equations are as follows:

$S_{0}=\frac{S_{5} \Theta_{1}+S_{6} \Theta_{2}}{S_{4}+S_{1} \Theta_{1}}$

$\Theta_{1}=\left(\frac{l}{\pi}\right)^{4} \sum_{m=1} \frac{1}{m^{4}+2 \beta m^{2}+\alpha}$

$\Theta_{2}=\left(\frac{l}{\pi}\right)^{6} \sum_{m=1} \frac{1}{m\left(m^{4}+2 \beta m^{2}+\alpha\right)}$

Here, $S_{0}, \alpha$, and $\beta$ can be expressed as follows:

$S_{0}=\sum_{m=1}\left(\frac{l}{m \pi}\right)^{2} a_{m}^{(1)} \cos m \pi$

$\alpha^{2}=\left(\frac{l}{\pi}\right)^{4} \frac{S_{2}}{S_{4}}$ 
$2 \beta=\left(\frac{l}{\pi}\right)^{2} \frac{S_{3}}{S_{4}}$

Hence, the closed-form solution of the interfacial stress can be obtained, and the equations can be expressed as follows:

$$
\tau_{(1)}= \begin{cases}\xi_{1}\left(\frac{l}{\pi}\right)^{3} \frac{S_{5}-S_{1} S_{0}}{S_{4}} g_{1}(x)+\xi_{1}\left(\frac{l}{\pi}\right)^{4} \frac{S_{6}}{S_{4}} g_{2}(x), & \alpha>\beta \\ \xi_{1}\left(\frac{l}{\pi}\right)^{3} \frac{S_{5}-S_{1} S_{0}}{S_{4}} g_{3}(x)+\xi_{1}\left(\frac{l}{\pi}\right)^{5} \frac{S_{6}}{S_{4}} g_{4}(x), & \alpha=\beta \\ \xi_{1}\left(\frac{l}{\pi}\right)^{3} \frac{S_{5}-S_{1} S_{0}}{S_{4}} g_{5}(x)+\xi_{1}\left(\frac{l}{\pi}\right)^{5} \frac{S_{6}}{S_{4}} g_{6}(x), & \alpha<\beta\end{cases}
$$

$$
\tau_{(2)}=\left(\frac{\xi_{2}}{\chi_{2} \xi_{1}}\right) \tau_{(1)}
$$

$\sigma_{(1)}=\frac{1}{\xi_{1}} \frac{d \tau_{(1)}}{d x}$

$$
\sigma_{(2)}=\frac{\eta_{2}}{\chi_{2}} \sigma_{(1)}
$$

$$
\text { Here, } g_{1}(\mathrm{x}), g_{2}(\mathrm{x}), g_{3}(\mathrm{x}), g_{4}(\mathrm{x}), g_{5}(\mathrm{x}) \text {, and } g_{6}(\mathrm{x}) \text { can be expressed as follows: }
$$

$$
\begin{aligned}
g_{1}(\mathrm{x}) & =\frac{\pi}{2 \sqrt{\alpha^{2}-\beta^{2}}\left(\sinh ^{2} \pi \gamma_{1}+\sinh ^{2} \pi \gamma_{2}\right)} \\
& \cdot\left[\cosh \frac{\pi \gamma_{1} x}{l} \sin \frac{\pi \gamma_{2} x}{l} \cdot \sinh \pi \gamma_{1} \cos \pi \gamma_{2}\right. \\
& \left.-\sinh \frac{\pi \gamma_{1} x}{l} \cos \frac{\pi \gamma_{2} x}{l} \cdot \cosh \pi \gamma_{1} \sin \pi \gamma_{2}\right]
\end{aligned}
$$




$$
\begin{aligned}
& +\frac{\pi}{2 \alpha^{2}} \cdot\left\{\frac{1}{\left(\sinh ^{2} \pi \gamma_{1}+\sinh ^{2} \pi \gamma_{2}\right)}\right. \\
& \cdot\left[\sinh \frac{\pi \gamma_{1} x}{l} \cos \frac{\pi \gamma_{2} x}{l} \cdot \sinh \pi \gamma_{1} \cos \pi \gamma_{2}\right. \\
& \left.\left.+\cosh \frac{\pi \gamma_{1} x}{l} \sin \frac{\pi \gamma_{2} x}{l} \cdot \cosh \pi \gamma_{1} \sin \pi \gamma_{2}\right]-\frac{x}{l}\right\}
\end{aligned}
$$

$g_{3}(\mathrm{x})=\frac{\pi^{2} x}{4 \gamma_{3} l} \frac{1}{\sinh \pi \gamma_{3}} \cosh \frac{\pi \gamma_{3} x}{l}-\frac{\pi^{2}}{4 \gamma_{3}} \frac{\operatorname{coth} \pi \gamma_{3}}{\sinh \pi \gamma_{3}} \sinh \frac{\pi \gamma_{3} x}{l}$

$$
+\frac{\pi}{2 \gamma_{3}^{4}} \frac{1}{\sinh \pi \gamma_{3}} \sinh \frac{\pi \gamma_{3} x}{l}+\frac{\pi^{2}}{4 \gamma_{3}^{3}} \frac{\operatorname{coth} \pi \gamma_{3}}{\sinh \pi \gamma_{3}} \sinh \frac{\pi \gamma_{3} x}{l}
$$

$g_{5}(\mathrm{x})=\frac{\pi}{4 \sqrt{\beta^{2}-\alpha^{2}}}\left[\frac{1}{\sinh \pi \gamma_{4}} \sinh \frac{\pi \gamma_{4} x}{l}-\frac{1}{\sinh \pi \gamma_{5}} \sinh \frac{\pi \gamma_{5} x}{l}\right]$

$$
\begin{aligned}
{ }_{6}(\mathrm{x}) & =\frac{\pi}{4 \sqrt{\beta^{2}-\alpha^{2}}}\left\{\frac{1}{\gamma^{2}}\left[\frac{1}{\sinh \pi \gamma_{5}} \sinh \frac{\pi \gamma_{5} x}{l}-\frac{x}{l}\right],\right. \\
& \left.-\frac{1}{\gamma^{2}}\left[\frac{1}{\sinh \pi \gamma_{4}} \sinh \frac{\pi \gamma_{4} x}{l}-\frac{x}{l}\right]\right\}
\end{aligned}
$$

where $\gamma_{1}, \gamma_{2}, \gamma_{3}, \gamma_{4}$, and $\gamma_{5}$ are parameters related to $\alpha$ and $\beta$. They can be expressed as follows:

$$
\begin{aligned}
& \gamma_{1}=\sqrt{\frac{\alpha+\beta}{2}} \\
& \gamma_{2}=\sqrt{\frac{\alpha-\beta}{2}} \\
& \gamma_{3}=\sqrt{\alpha}
\end{aligned}
$$


$\gamma_{4}=\sqrt{\beta+\sqrt{\beta^{2}-\alpha^{2}}}$

$\gamma_{5}=\sqrt{\beta-\sqrt{\beta^{2}-\alpha^{2}}}$

\section{INTERFACIAL STRESS TESTS OF CFRP-CONCRETE SPECIMENS}

The theoretical model of the proposed CFRP-reinforced concrete beams was experimentally verified. The beam specimen was designed according to the box-girder force analysis results, and the test setup and instrumentation were based on the actual bridge load boundary conditions.

\subsection{Experimental specimens and test setup}

\subsubsection{Specimen description}

According to the analysis results of the original bridge structure (Figure 2(a)), a CFRP-strengthened concrete beamslab specimen was designed. The test specimen had a reinforced concrete beam-slab structure with a rectangular crosssection. Its dimensions were $\mathrm{I} \times \mathrm{b} \times \mathrm{h}=4000 \mathrm{~mm} \times 650 \mathrm{~mm} \times 280 \mathrm{~mm}$. The concrete grade of the test beam was C45, and the reinforcement in the tension and compression zones was HRB400 rebar with a diameter of $12 \mathrm{~mm}$. The test beam reinforcement layout is shown in Figure 4.

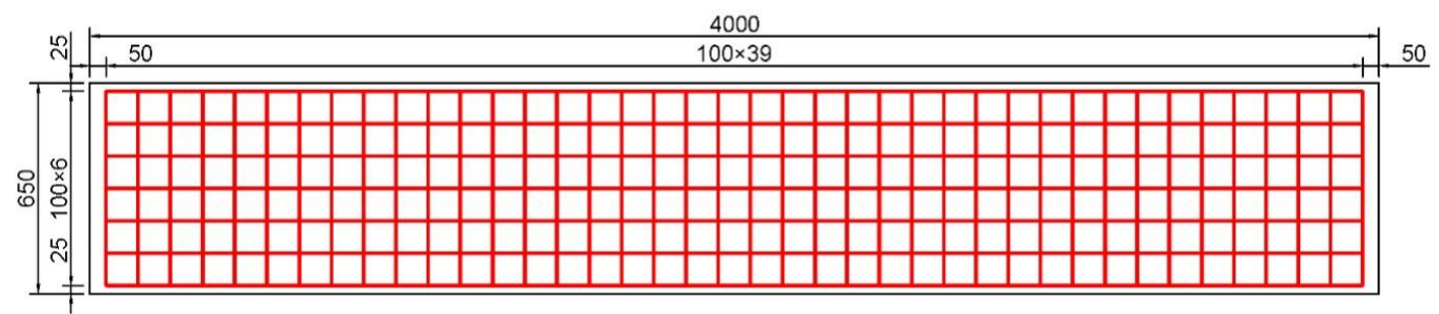

(a) plan layout

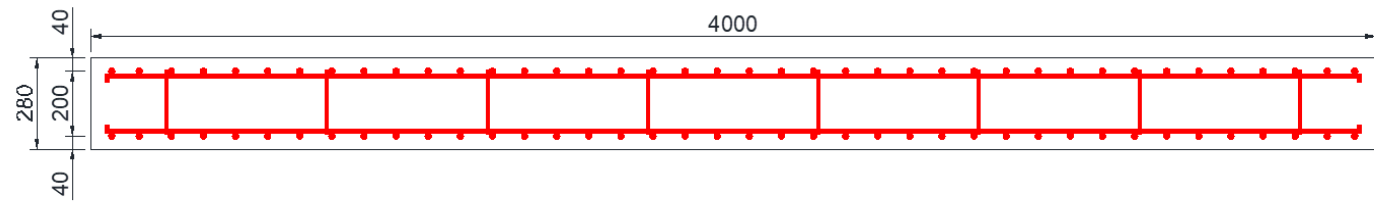

(b) vertical section

650

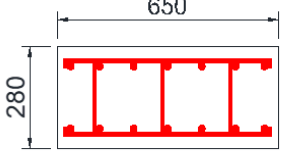

(c) cross section

Figure 4: Specimen dimensions and reinforcement layout (unit: $\mathrm{mm}$ ).

To strengthen the test beam, three CFRP plates with a certain length were pasted on its top surface, as shown in Figure 5(a). The cross-sectional size of the CFRP plate was $\mathrm{h} \times \mathrm{b}=1.4 \mathrm{~mm} \times 100 \mathrm{~mm}$. After the test beam was reinforced with the CFRP plate, its top surface was paved with stone mastic asphalt to simulate the high-temperature effect and dead weight of the asphalt layer, as shown in Figure 5(b). 


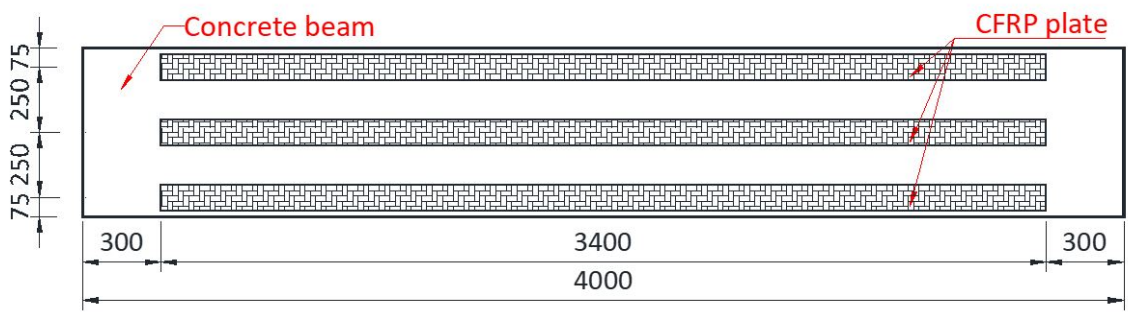

(a) Arrangement of CFRP plate

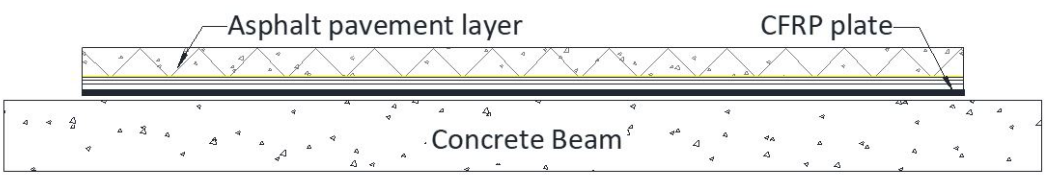

(b) Asphalt layer pavement on the beam specimen

Figure 5: Specimen strengthening with the CFRP plate and asphalt layer paving construction.

In the test, two sets of specimens (a total of four specimens) were investigated. The FDBL series, which had one test beam, was used for comparison. The FX series contained three test beams (i.e., FX-1, FX-2, and FX-3) whose specimens were strengthened with CFRP plates. The strengthening scheme is shown in Figure 5(a).

\subsubsection{Material property}

For this test, the materials used in the test beam mainly included the CFRP plate, concrete, steel bars, and adhesives. The elastic moduli of the C45 concrete and steel bars were 33.5 and 2.5-2.6 GPa, respectively. The main performance parameters of the CFRP plate and adhesive are presented in Table 2.

Table 2: Measured performance indices of the CFRP plate and adhesive.

\begin{tabular}{cccc}
\hline Material type & CFRP & Material type & K-801 \\
\hline Tensile strength (MPa) & $2300-2400$ & Tensile strength (MPa) & $25-26$ \\
Tensile modulus (GPa) & $150-155$ & Tensile modulus of elasticity (MPa) & $2500-2600$ \\
Elongation at break (\%) & $1.4-1.42$ & Bending strength (MPa) & $30-32$ \\
& & Compressive strength (MPa) & $70-75$ \\
\hline
\end{tabular}

\subsubsection{Test procedure and instrumentation}

The beam specimens were simply supported at the midspan of the test beam. Two bearings were arranged at $400 \mathrm{~mm}$ on both sides of the beam midspan, and the loading positions were at the two ends of the beam. Moreover, displacement meters were arranged at the beam ends and midspan positions to measure the beam deformation during the test. The test setup is shown in Figures 6(a) and (b).

The interfacial stress between the CFRP plate and the concrete was the focus of this study; hence, it was necessary to examine the strain distribution at the CFRP-concrete interface. However, measuring the strain at the interface layer during the test is difficult. It can be calculated through a certain equivalent transformation of the CFRP-plate strain. To measure the strain, a part of the asphalt layer was removed before the test. Considering the symmetry of the structure, a half-beam-long portion of the asphalt layer was removed from each of the CFRP plates. Strain gauges were arranged on the outer layers of the three CFRP plates; the layout of these gauges on the CFRP plate is shown in Figure 6(c).

A steel bearing plate was arranged at each loading position to prevent stress concentration. A vertical force of $300 \mathrm{kN}$ was simultaneously applied to each steel bearing plate. During the loading process, the load was gradually applied for preventing the excessive deformation of the specimen, as shown in Figure 6(d). 


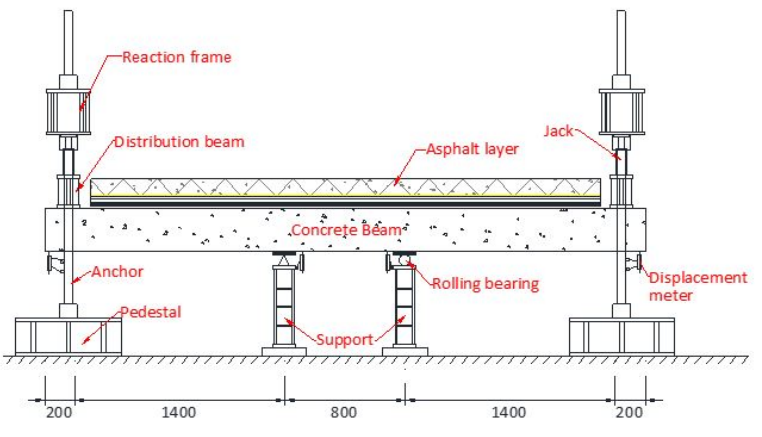

(a) front view

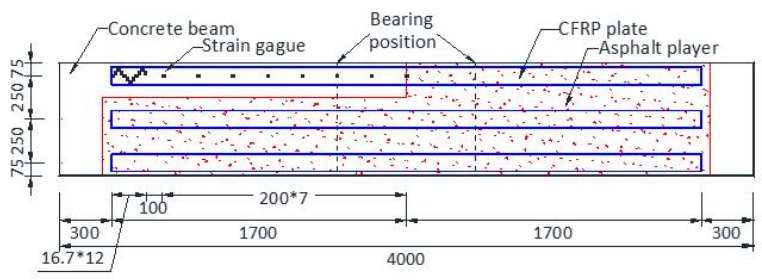

(c) arrangement of strain gauges

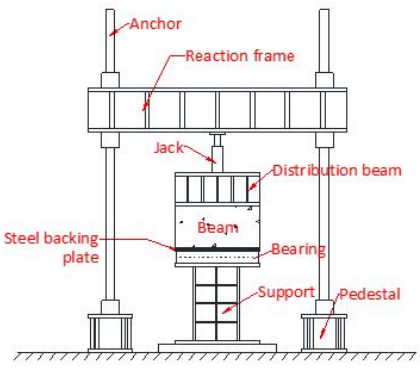

(b) side view

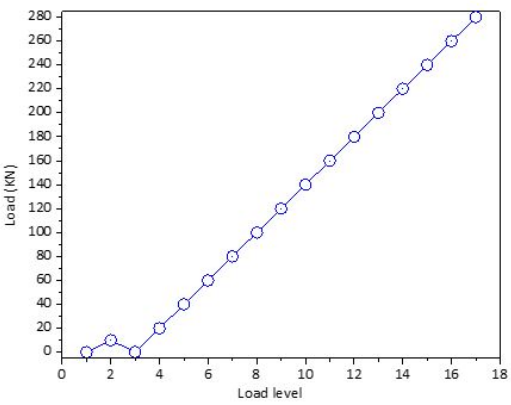

(d) loading pattern

Figure 6: Test setup and loading process.

\subsection{Failure characteristics and observations}

3.2.1 Debonding failure phenomenon and bearing capacity

The failure form and bearing capacity of the specimen were investigated during the test. The common failure modes of CFRP-strengthened concrete beams are as follows.

a) Plate-end debonding failure: when the interfacial stress at the end of the CFRP plate exceeds the ultimate bearing capacity of the material, plate-end interfacial debonding failure is expected to occur. Thus, this type of failure mode is usually caused by high interfacial stress.

b) Intermediate crack-induced debonding: various flexural cracks and flexure-shear cracks typically appear at the midspan of the beam. Usually, intermediate cracks lead to this type of failure mode.

The failure patterns of different specimens were examined during the test, as shown in Figure 7. 
(a) Specimen FDBL

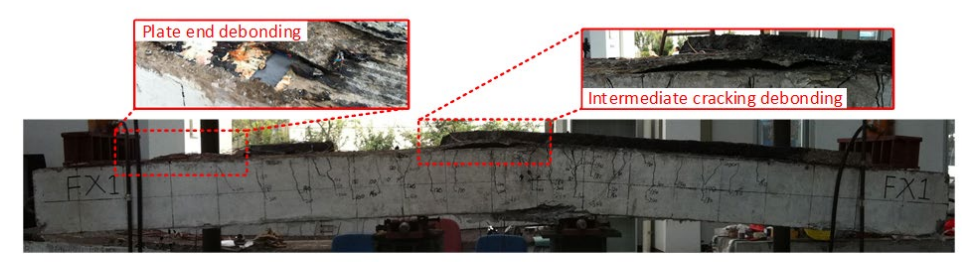

(b) Specimen FX-1

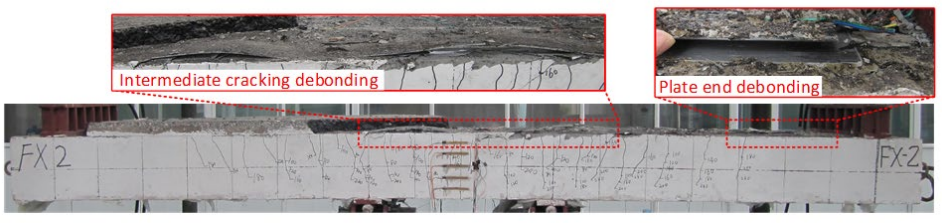

(c) Specimen FX-2

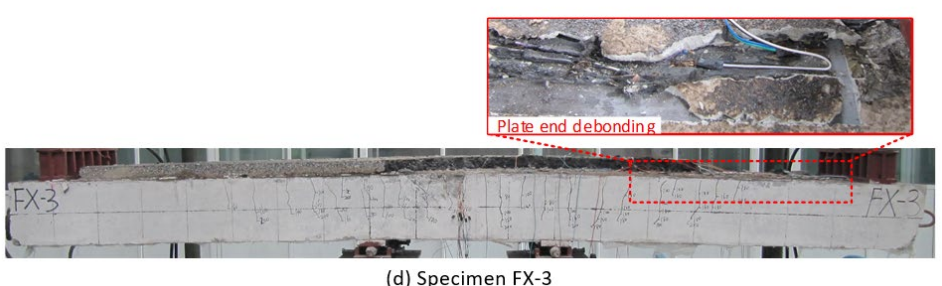

Figure 7: Cracking pattern and failure characteristics of specimens.

As shown in Figure 7, the failure mode of the FDBL specimen was mainly the ductile deformation of the tensile steel bar, and those of the FX series specimens were concrete cracking in the negative-flexural moment zone and debonding failure of the CFRP plate.

For specimen FX-1, distinct intermediate crack-induced debonding failure and plate-end debonding failure were observed. After the beam was unloaded, the CFRP plate of the test beam exhibited a significant cumulative deformation, and a void phenomenon occurred between the CFRP plate and the concrete at the midspan.

The failure mode of specimen FX-2 was similar to that of specimen FX-1. However, the debonding failure of specimen FX-2 was interfacial debonding failure between the CFRP plate and adhesive layer, whereas that of specimen FX-1 was the debonding failure of the surface layer concrete cover around the interface layer.

The debonding failure of specimen FX-3 was not observed at the midspan of the test beam and only occurred at the plate end.

Compared with the plastic deformation of specimen FX-1, those of FX-2 and FX-3 were smaller. This may have been due to problems in the construction quality during the pasting process and the high-temperature effect during asphalt paving. The foregoing also resulted in debonding failure or voiding at some positions of the interface layer before the test. This preexisting debonding failure prevented the full exertion of the tensile effect of the CFRP plate.

For comparison, the moments in the specimens at different loading stages were calculated. The calculation results and debonding patterns are presented in Table 3.

Table 3: Bearing capacity and debonding form (unit: $\mathrm{kN} \cdot \mathrm{m}$ ).

\begin{tabular}{cccccc}
\hline Number & Mcr & My & Mdb (Mu) & Mdb (Mu)/ My & Debonding pattern \\
\hline FDBL & 42 & 84 & 98 & 1.167 & $/$ \\
FX-1 & 42 & 140 & 168 & 1.200 & ICD \& PED \\
FX-2 & 42 & 154 & 168 & 1.091 & ICD \& PED \\
FX-3 & 42 & 140 & 154 & 1.100 & PED \\
\hline
\end{tabular}

Notes: Mcr and My represent the cracking moment and flexural yield moment of the specimens, respectively. Mu represents the ultimate moment of the unstrengthened specimen, and Mdb represents the debonding moment of the strengthened specimen. Abbreviations: Mcr, cracking moment of specimen; My, flexural yield moment of specimen; Mu, ultimate moment of un-strengthened specimen; Mdb, debonding moment of strengthened specimen; ICD, intermediate crack-induced debonding; PED, plate-end debonding. 
As indicated by Table 2, the average ultimate bearing capacity of the strengthened specimen was $66.7 \%$ higher than that of the un-strengthened specimen. It can be concluded that the CFRP-strengthened method can effectively improve the ultimate bearing capacity and delay the occurrence of debonding failure.

\subsubsection{CFRP-plate strain}

The strain distributions in the CFRP plates under different loads were measured using the strain gauges on the CFRP plates. The strain distributions in the different specimens are shown in Figure 8.

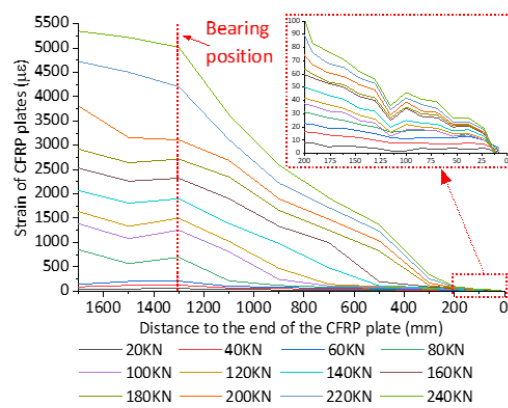

(a) Specimen FX-1

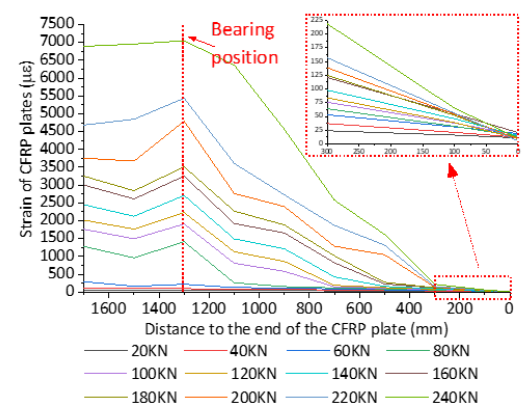

(b) Specimen FX-2

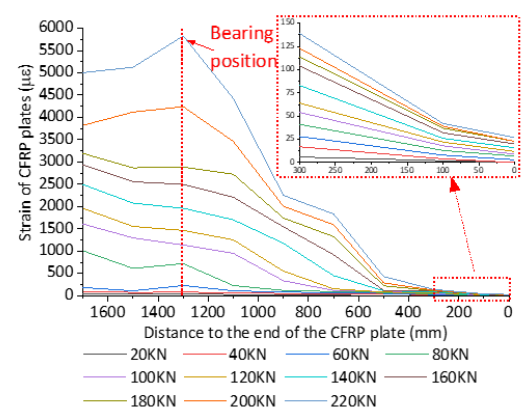

(c) Specimen FX-3

Figure 8: Strain distributions in the CFRP plates under different loads.

As shown in Figure 8, the strain change in the CFRP plate was not distinct when the loading level was low. As the loading level increased, the strain distribution in the CFRP plate exhibited nonlinearity. When the loading level was high, the CFRP plates exhibited high and low strain values at the midspan and end positions of the plates, respectively. When the loading level was approximately $220 \mathrm{kN}$, the CFRP strain $1400 \mathrm{~mm}$ from the plate end exceeded that at the adjacent position. The expansion of cracks in the concrete beam was the main reason for this situation. Near the midspan, a highconcrete stress area existed around the crack position. The CFRP-plate strain was also increased by the bonding force between the CFRP plate and the concrete.

\subsubsection{Interfacial shear stress between CFRP plate and concrete}

Rahimi et al. (2001) proposed a method based on force balance for calculating the shear stress of the CFRP platereinforced concrete structure interface, and the interfacial stress between the CFRP plate and the concrete can be determined using the adjacent strain value in the CFRP plate.

The interfacial stress can be calculated using the strain values measured by the adjacent strain gauges, via the following equation.

Assume that the adjacent strain values in the CFRP plate are $\Delta \varepsilon_{i}$ and $\Delta \varepsilon_{j}$. The longitudinal force difference in the CFRP plate between both adjacent strain measurement points can be calculated using Eq. (17):

$\Delta F_{t}=\operatorname{Ebt}\left(\Delta \varepsilon_{j}-\Delta \varepsilon_{i}\right)$ 
where $\Delta F_{t}$ represents the longitudinal force difference between both aforementioned points within the length $\Delta l ; E$ represents the elastic modulus of the CFRP plate; $b$ and $t$ represent the width and thickness of the CFRP plate, respectively; and $\Delta \varepsilon_{i}$ and $\Delta \varepsilon_{j}$ represent the CFRP-plate strains measured by the $\mathrm{i}^{\text {th }}$ and $\mathrm{j}^{\text {th }}$ strain gauges, respectively.

The interfacial shear force between the CFRP plate and the concrete can be calculated using the following equation:

$\Delta F_{l}=\tau_{l} b \Delta l$,

where $\Delta F_{l}$ represents the difference in the interfacial shear force within the length $\Delta l$, and ${ }^{\tau_{l}}$ represents the interfacial shear stress between the CFRP plate and the concrete in the length $\Delta l$.

According to the principle of force balance, $\Delta F_{t}$ in Eq. (17) should be equal to $\Delta F_{l}$ in Eq. (18). The following equation can be obtained:

$\tau_{l}=\left(\Delta \varepsilon_{l} / \Delta l\right) E t$

where the obtained interfacial shear stress $\left({ }^{\tau_{l}}\right)$ is the average interfacial shear stress in the length $\Delta l$, which approximately corresponds to the center positions of the adjacent strain gauges.

Rahimi et al. (2001) proposed a calculation method for the shear stress at the interface between the CFRP plate and the concrete. The calculation results for the interfacial shear stress of each specimen are shown in Figure 9.

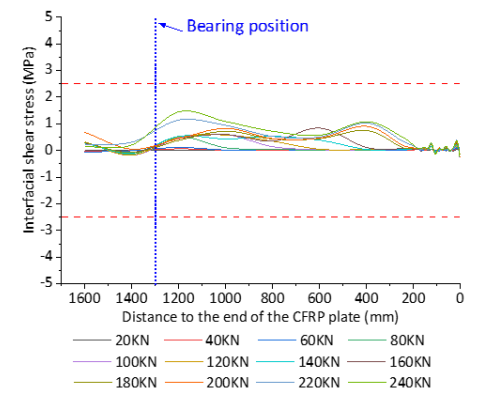

(a) Specimen FX-1

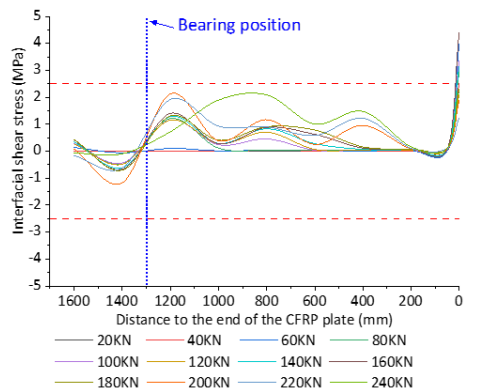

(b) Specimen FX-2

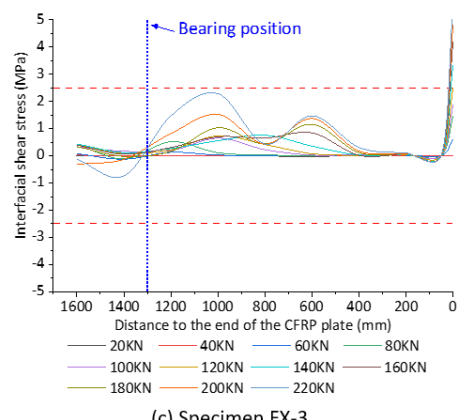

(c) Specimen FX-3

Figure 9: Interfacial shear stresses in different specimens.

Considering that the bonding strength of the adhesive layer is high, the interfacial debonding failure between the CFRP plate and concrete is generally indicated by the debonding failure of the concrete surface layer. It can be considered that interfacial debonding failure has occurred when the interfacial shear stress exceeds the concrete tensile strength. Therefore, the calculated interfacial shear stress was compared with the concrete ultimate bearing capacity in tension, as shown in Figure 9. When the loading level was low, the interfacial shear stress was relatively low. The interfacial shear stress fluctuated as the load increased, but it did not reach the tensile strength of concrete materials.

For specimens FX-2 and FX-3, the position where the interface shear stress curve changed suddenly was at the end of the CFRP plate, and the interfacial shear stress exceeded the concrete bearing capacity in tension. It is considered that 
plate-end debonding failure occurred at this position in the CFRP plate, corresponding to the failure mode of the specimen during the test (Figures 7(b)-(d)).

However, for specimen FX-1, the interfacial shear stress at the end of the CFRP plate fluctuated only slightly under the different loading levels. Considering the failure form of the specimen shown in Figure 7(a), a large plastic deflection was observed after the loading process. The CFRP plate was considered to be not fully effective, and voiding of the interface layer may have existed before the test. The CFRP plate of specimen FX-1 was in the free end state during the test. Therefore, no distinct deformation occurred in this part of the CFRP plate, and the interfacial shear stress in this segment could not be accurately measured.

\section{ANALYSIS OF RESULTS AND APPLICATIONS}

\subsection{Comparison between analysis and test results}

The interfacial stresses calculated using the theoretical model and measured in the flexural beam tests are compared in Figure 10.

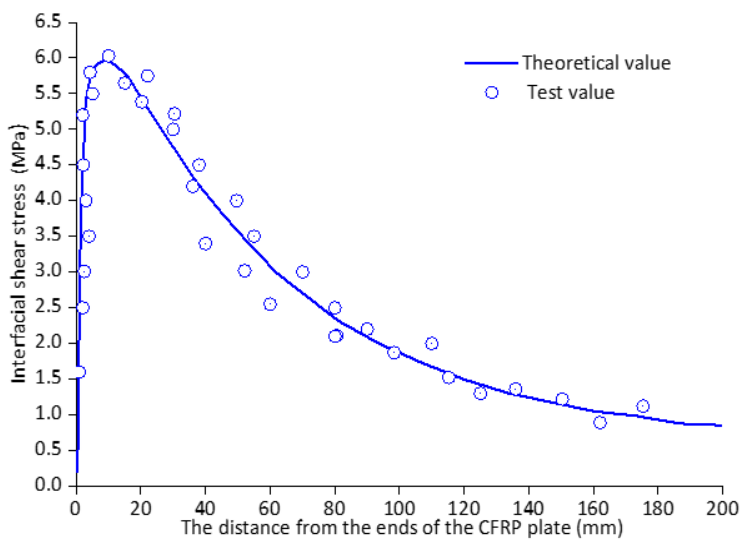

(a) Interfacial shear stress

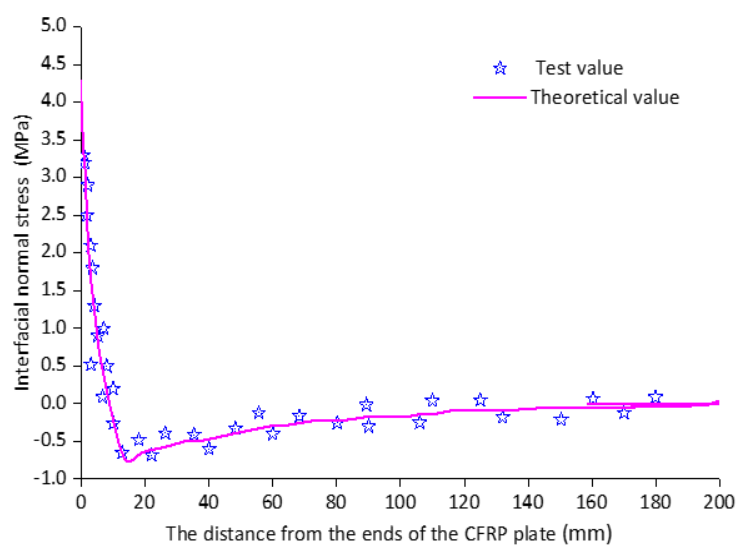

(b) Interfacial normal stress

Figure 10: Comparison between theoretical and experimental values.

As shown, the distribution of the experimental values agreed well with the theoretical curve, indicating that the proposed interfacial-stress calculation model is reliable.

Similar to the test results, the shear stress of the CFRP-concrete interface at the end of the CFRP plate changed significantly near the end position. The difference between the calculation and test rests for the end position was small.

The peak of the interface normal stress in the CFRP plate-concrete interface appeared at the end position. The interface layer at this position was subject to tensile stress and prone to debonding failure. 
Along the longitudinal direction of the CFRP plate, the interfacial normal stress rapidly decreased from the end position and became negative. At this position, the interface was under pressure, preventing debonding failure. The interface normal stress approached zero with the increasing distance from the end of the CFRP plate.

\subsection{Application to structural calculation}

According to the general codes for the design of highway bridges and culverts adopted in China, two types of vehicleload grades are employed in bridge design: highway classes I and II. Lane loading can be converted into a uniform load (qk) and concentrated force (pk). When the load grade is highway class I, the standard value of the uniform load is $10.5 \mathrm{kN} / \mathrm{m}$, and that of the concentrated force is based on the bridge span. When the calculation span is $<5 \mathrm{~m}$ and $>50 \mathrm{~m}$, the load is considered to be 270 and $360 \mathrm{kN}$, respectively. For other spans, the linear-interpolation method is employed to obtain the load value. When the vehicle-load grade is highway level II, the lane loading magnitude adopted is 0.75 times that specified for highway class I.

Accordingly, the lane loading in the general specifications was converted into the load of the CFRP plate- reinforced box-girder roof in this study. The maximum and minimum values of the concentrated load specified by the lane loading of highway classes I and II were selected for the calculation. The calculation results for the interfacial stress distributions under different loading levels are shown in Figure 11.

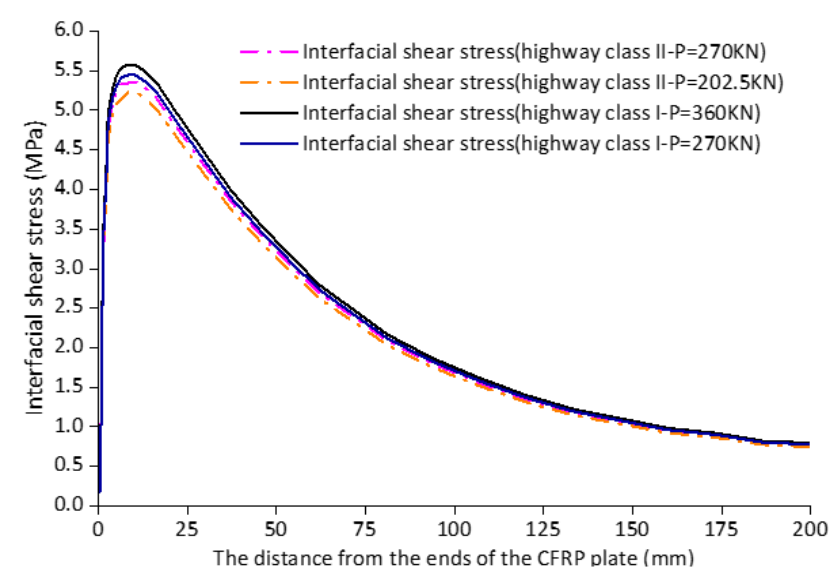

(a) Theoretical interfacial shear stress

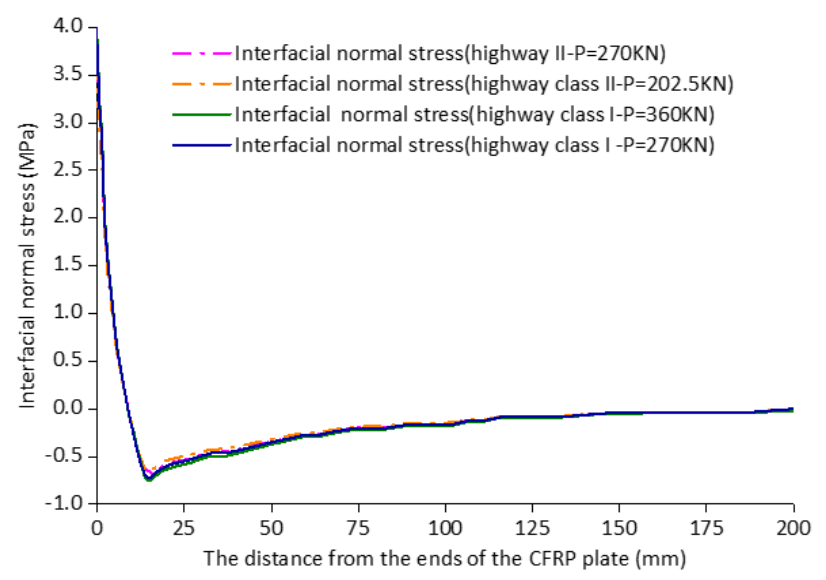

(b) Theoretical interfacial normal stress

Figure 11: Theoretical values of the interfacial stress under different bridge loads.

As shown in Figure 11 (a) and (b), under different loads (highway class I-P=270kN, I-P=360kN, II-P= 202.5kN, II$\mathrm{P}=270 \mathrm{kN}$ ), the interface normal stress and shear stress appear peak near the end of CFRP plate peeling, and the interface normal stress and shear stress near the end of CFRP plate peeling approach to zero at the end of CFRP plate peeling. The variation range of the shear stress curve data on the peak value is relatively significant compared with that of the normal stress data, and the peak value of the normal stress curve data only fluctuates within a small range. The comparison of the peak value is shown in Table 4. 
Table 4: Peak value of theoretical interface shear stress and normal stress under different highway grades.

\begin{tabular}{ccccc}
\hline Load level (KN) & I-P=270 & II-P=202.5 & I-P=360 & II-P=270 \\
\hline Theoretical interfacial shear stress (MPa) & 5.44 & 5.23 & 5.58 & 5.37 \\
Theoretical interfacial normal stress(MPa) & -0.71 & -0.65 & -0.75 & -0.70 \\
\hline
\end{tabular}

Note: The minus sign indicates the opposite direction of the hypothesis

At the same time, it can be seen from Figure (a) and (b) that under four different loads (highway class I-P $=270 \mathrm{kN}$, $I-P=360 \mathrm{kN}, I I-P=202.5 \mathrm{kN}, I I-P=270 \mathrm{kN})$, for shear stress and normal stress in Figure (a), the overall fluctuation trend of the four curves in each figure is similar. To further scientific analysis of the phenomenon, we with no significant difference between every four sets of data for the assumptions, the significance level of $0.01 \mathrm{a}$, respectively according to the theory of shear stress and normal stress of the four groups of data analysis of variance, can draw the following conclusions: (1) for shear stress data by variance analysis, the parameter values of P-value is 0.97 , the parameter value $F 0.09$, parameter value $\mathrm{F}$ crit is 3.9. (2) for normal stress data by variance analysis, the $\mathrm{p}$-value of the parameter is 0.99 , the parameter value $F$ is 0.003 , and the parameter value $F$ crit is 3.8 , as shown in Tables 5 and 6.

Table 5: Analysis of variance of theoretical interfacial shear stress data under different highway grades.

\begin{tabular}{ccccccc}
\hline Source & SS & df & MS & F & P-value & F crit \\
\hline Between the group & 0.74 & 3 & 0.24 & 0.09 & 0.97 & 3.9 \\
Within the group & 384.71 & 140 & 2.74 & - & - & - \\
The total & 385.48 & 143 & - & - & - \\
\hline
\end{tabular}

Notes: SS represents the sum of squares in the table; Df stands for degrees of freedom; MS is the mean square; $F$ is the test statistic; P-value is the number of the null hypothesis; $\mathrm{F}$ crit is the critical value.

Table 6: Analysis of variance of theoretical interface normal stress data under different highway grades.

\begin{tabular}{ccccccc}
\hline Source & SS & df & MS & F & P-value & F crit \\
\hline Between the group & 0.01 & 3 & 0.003 & 0.003 & 0.99 & 3.8 \\
Within the group & 247.4 & 200 & - & - & - & - \\
The total & 247.41 & 203 & - & - & - \\
\hline
\end{tabular}

Through the comparison of these parameters, the relationship can be obtained: P-value $>a, F<F$ crit. According to the ANOVA evaluation criteria, the null hypothesis can be accepted and no significant difference can be found between every four groups of data under different stresses. Therefore, in each graph, the overall fluctuation trend of the four curves is similar and the range of change is not large. According to the above analysis, the distribution characteristics of the model are relatively stable and can be applied to engineering practice.

\section{CONCLUSIONS}

A closed interfacial-stress calculation model was developed by studying the interfacial stress between a CFRP plate and concrete and the load characteristics of the bridge. To verify the reliability of the theoretical model, experimental tests were performed. According to the results, the following conclusions are drawn.

1. With the higher-order analytical solutions of the interfacial-stress calculation model, the approximation used in the previous solution of the simply supported beam is avoided.

2. The concentrated load, uniform load, and flexural moment were considered in the proposed model. Different loading conditions can improve the usefulness of theoretical models in practical engineering.

3. The test beams were designed according to the box girder. The intermediate cracking debonding failure and plate-end debonding failure of the test beam can be observed. The interfacial shear stress was obtained according to the adjacent strain in the CFRP plate, and the high-interfacial stress region coincided with the occurrence of the debonding phenomenon in the test beams.

4. The interfacial stress obtained via the flexural tests agreed well with the calculation results of the theoretical model, hence validating the theoretical model. 
5. The interfacial-stress curve calculated using the theoretical model according to the different loading levels specified in the code was consistent with the interfacial-stress curve obtained from the test.

6. The change response sensitivity of the interfacial shear stress was higher than that of the interfacial normal stress under different loading levels and according to ANOVA, the overall fluctuation trend of the four curves in each graph is similar, and the range of change is small. The distribution characteristics of the model are relatively stable and can be applied to engineering practice

\section{ACKONWLEDGEMENTS}

This work was financially supported by the Brand Professional Funding Project of Jiangsu Province (Grant No. PPZY2015B143), the National Natural Science Foundation of China (Grant No. 51508368), the Science and Technology Project of Housing and Urban-Rural Development Department of Jiangsu Province(Grant No.2019ZD010), the Postgraduate Research \& Practice Innovation Program of Jiangsu Province (Grant No. SJCX18_0879), and Key Project of Innovation and Entrepreneurship of College students in Jiangsu Province (Grant No. 201810332019Z).

Author Contributions: Conceptualization, X Yuan, C Sun and Q Li; Methodology, X Yuan and C Sun; Investigation, X Yuan, C Sun, Q Li, C Zhu, and S Tang; Writing - original draft, X Yuan, C Sun and Q Li; Writing - review \& editing, X Yuan, C Sun, $Q$ Li, C Zhu; Funding acquisition, X Yuan, C Sun; Resources, X Yuan and C Zhu; Supervision, X Yuan, C Sun and S Tang.

Editor: Rogério José Marczak

\section{REFERENCES}

A. Hosseini, E. Ghafoori, R. Al-Mahaidi, X-L. Zhao, M. Motavalli. (2009) Strengthening of a 19th-century roadway metallic bridge using nonprestressed bonded and prestressed Unbonded CFRP plates. Construction and Building Materials 209: 240 -259.

B. Kromoser, P. Preinstorfer, J. Kollegger. (2019) Building lightweight structures with carbon fiber-reinforced polymerreinforced ultra-high-performance concrete. fib. International Federation for Structural Concrete 20: 730-744.

C.J. Burgoyne. (2004) Does FRP have an economic future? Advanced Composite Materials in Bridges and Structures, Calgary, Alberta, July 20-23.

C. Czaderski, M. Motavalli. (2007) 40-Year-old full-scale concrete bridge girder strengthened with prestressed CFRP plates anchored using gradient method. Composites Part B 38: 878-886.

H. Rahimi, A. Hutchinson. (2001) Concrete beams strengthened with externally bonded FRP plates. Journal of Composites for Construction 5(1):44-56.

J.M. Gallego, J. Michels, C. Czaderski. (2017) Influence of the asphalt pavement on the short-term static strength and longterm behaviour of RC slabs strengthened with externally bonded CFRP strips. Engineering Structures 150: 481-496.

J.M. Gallego, C. Czaderski, M. Breveglieri, J. Michels. (2018)Fatigue behaviour at elevated temperature of RC slabs strengthened with EB CFRP strips. Composites Part B 141: 37-49.

X.Z. Lu, L.P. Ye, J.G. Teng, J.J. Jiang. (2005) Meso-scale finite element model for FRP sheets/plates bonded to concrete. Engineering Structures 27: 564-575.

S.T. Smith, J.G. Teng. (2002) FRP-strengthen RC beams. I: review of debonding strengthen models. Engineering Structure24(4): 385-395.

W. Sun, X. Peng, H.F. Liu, H.P. Qi. (2017a) Numerical studies on the entire debonding propagation process of FRP strips externally bonded to the concrete substrate. Construction and Building Materials 149: 218-235.

W. Sun, X. Peng, Y. Yu. (2017b) Development of a simplified bond model used for simulating FRP strips bonded to concrete. Composite Structures 171: 462-472.

E. Ferrier, L. Michel, B. Jurkiewiez, P. Hamelin. (2011) Creep behaviour of adhesives used for external FRP strengthening of RC structures. Construction and Building Materials,25: 461-467. 
E. Ferrier, O. Rabinovitch, L. Michel. (2016) Mechanical behavior of concrete-resin adhesive FRP structural assemblies under low and high temperatures. Construction and Building Materials, 127: 1017-1028.

E. Ferrier, A. Agbossou. (2017) Temperature effects on the shear capacity of external bonded fiber reinforced polymer on concrete. Construction and Building Materials 152: 333-344.

X.M. Wang, M. Petrů. (2019) Mode I fracture evaluation of CFRP-to-concrete interfaces subject to aggressive environments agents: Freeze-thaw cycles, acid, and alkaline solution. Composites Part B 168: 581-88.

H. Rahimi, A. Hutchinson. (2001) Concrete beams strengthened with externally bonded FRP plates. Journal of Composites for Construction 5(1):44-56. 